\title{
Travaux parlementaires autour de la LAMal: le suspense perdure...
}

Yves Guisan, Vice-président FMH

\section{Arrêté fédéral urgent sur le financement hospitalier}

La première urgence de la commission de sécurité sociale et de santé du Conseil national lors de sa dernière séance des 10,11, et 12 avril dernier, a été de régler le sort de l'arrêté fédéral urgent concernant la participation des cantons au financement de l'hospitalisation privée et semi-privée dans les établissements publics conformément au jugement du Tribunal fédéral des assurances. Ce dernier s'est prononcé en fonction des dispositions de l'art. 49 \$1 de la LAMal qui ne concerne que les établissements publics ou subventionnés. En conséquence la proposition du Conseil des Etats pour permettre aux cantons d'adapter progressivement leur participation estimée à quelques 600 millions d'ici 2004 ne visait à rien d'autre que de mettre en place des modalités d'application acceptables.

\section{Egalité cliniques privées - hôpitaux publics}

Il est vrai que cette manière de faire ne résout que partiellement une discrimination criante. Les cliniques privées sont en effet exclues, alors que les patients qui y sont traités sont également des contribuables et des cotisants de l'assurance obligatoire. De l'avis d'une plus que courte majorité de la commission (10 voix contre 10 départagée par son président) ils ont donc tout autant droit à leur part cantonale de l'assurance de base que ceux des divisions privées et semiprivées des hôpitaux publics. Aussi a-t'elle étendu ces dispositions de financement à tous les hôpitaux qui figurent sur la liste cantonale de planification.

\section{Court-circuiter le processus législatif et démocratique?}

Ceci n'est malheureusement pas sans problèmes. Non seulement il en coûtera quelques 200-300 millions de plus aux cantons, mais surtout il aurait été une nouveauté juridique et institutionnelle pour le moins déroutante qu'un arrêté fédéral urgent, valable 1 année et non soumis au référendum, anticipe sur une modification de la loi avant que celle-ci n'ait été décidée selon la procédure législative habituelle. La révision de la LAMal en cours envisage effectivement de mettre à égalité cliniques privées et établissements publics ou subventionnés en introduisant un financement à la prestation (forfaits type DRG). Mais rien n'est encore fait. Anticiper sur la procédure de la sorte revient tout simplement à court-circuiter le processus législatif et démocratique en cours. Il pourrait donc créer un précédent lourd de signification, a fortiori s'il devait être étendu par la suite à d'autres situations délicates.

\section{La commission dégage en corner ...}

La commission a donc décidé de renoncer au caractère urgent de l'arrêté et de le soumettre à la procédure ordinaire y compris le référendum facultatif... En attendant les cantons peuvent respirer encore d'un souffle, et les cliniques privées continuer à piétiner dans l'attente d'une décision qui ne pourra pas entrer en vigueur en 2002 et sera retardée au moins d'une année, à moins que le Conseil national n'en reste à la proposition du Conseil des Etats au cours de la prochaine session de juin prochain ...

\section{Oui au financement hospitalier, mais attention aux DRG!}

Pour le reste la commission a surtout cherché à planifier et organiser son travail à l'avenir. Elle considère que le projet de financement hospitalier est pratiquement prêt et ne devrait pas occasionner de difficultés particulières en dehors de quelques amendements (mise en place de critères de planification fédéraux par exemple). Rendu attentive aux dérapages en matière de cost weight de l'expérience vaudoise AP-DRG en cours, l'administration fédérale fixera son choix sur une classification des patients qui évite dans toute la mesure les effets pervers de ces modalités d'indemnisation. Les forfaits doivent tenir compte de la mission hospitalière telle que 
définie par la planification (service d'urgence $24 \mathrm{~h}$ sur $24 \mathrm{~h}$, maintien d'un établissement de rentabilité aléatoire dans une région périphérique éloignée, mission spécialisée particulière, etc.) et ne doivent pas donner lieu à une sélection indue des risques (transfert des cas non rentables dans les hôpitaux cantonaux et universitaires ...). Par contre tout système tarifaire a ses imperfections et il faudra en tolérer un certain nombre.

\section{Prolongations certaines}

Les autres aspects de la révision, passage à un financement moniste, suppression de l'obligation de contracter, financement social des primes (plafonnement à $8 \%$ du revenu) posent d'innombrables questions sans réponse actuellement. La commission est d'avis qu'il n'est pas possible d'inclure dans la loi des réformes sous forme de postulats de principe sans en connaître les effets potentiels dans toute la mesure du possible. La révision de la loi doit être sérieuse, crédible, et ne pas nécessiter de nouveaux rafistolages à peine entrée en vigueur comme cela a été le cas jusqu'à présent. Ces projets doivent donc être affinés avec des simulations et des projections précises. Il est certain que ces exigences ne permettront pas de voir ces objets traités lors de la prochaine session: Ils devront être reportés jusqu'en septembre au moins. Le mandat de la sous-commission LAMal a donc été renouvelé afin de présenter prochaine- ment les différents scénarios à prendre en considération quant à la levée de l'obligation de contracter.

\section{TARMED au centre d'une décision de principe}

Ils sont au nombre de quatre: le statu quo avec TARMED, la proposition du Conseil des Etats avec suppression complète de l'obligation de contracter, celle du Conseil fédéral avec suppression de l'obligation de contracter pour les consœurs/confrères de plus de 65 ans seulement, et la proposition socialiste de ne la supprimer que pour les spécialistes, mais pas pour les médecins de premier recours. Mais ces trois dernières possibilités ne pourront pas inclure également TARMED et ses annexes négociées. Les membres de la sous-commission ont en effet pris connaissance avec la plus grande surprise de la possibilité de dénonciation prévue par la convention-cadre TARMED en cas de suppression de l'obligation de contracter sous une forme ou sous une autre pendant la phase d'introduction du tarif (art. 18, pt. 6). La commission d'abord et le Conseil national ensuite sont donc appelés à prendre une décision de principe fondamentale: les coûts de la santé doivent-ils être maîtrisés par la dérégulation de l'assurance sociale obligatoire ou plutôt par des accords négociés entre partenaires en fonction des objectifs de la loi? 